\title{
TNF-Alpha in Peripheral Neuropathy Patients with Impaired Glucose Regulation
}

\author{
Xia Li, ${ }^{1,2,3}$ Ju Zhu, $^{2,3}$ Na Liu, ${ }^{2,3}$ Jie Liu, ${ }^{2,3}$ and Zhecheng Zhang ${ }^{2,3}$ \\ ${ }^{1}$ The Third Central Clinical College of Tianjin Medical University, Tianjin, China \\ ${ }^{2}$ Department of Neurology, Tianjin Third Central Hospital, Tianjin, China \\ ${ }^{3}$ Tianjin Key Laboratory of Artificial Cell, Tianjin, China
}

Correspondence should be addressed to Zhecheng Zhang; zzc0912@126.com

Received 16 August 2016; Revised 23 October 2016; Accepted 9 November 2016; Published 30 January 2017

Academic Editor: Norman Cameron

Copyright (C) 2017 Xia Li et al. This is an open access article distributed under the Creative Commons Attribution License, which permits unrestricted use, distribution, and reproduction in any medium, provided the original work is properly cited.

\begin{abstract}
Impaired glucose regulation (IGR) is the prestate of diabetes; about 1/3 of IGR patients will develop to diabetes finally. In this study, we investigated the serum tumor necrosis factor-alpha (TNF- $\alpha$ ) and interleukin-6 (IL-6) levels in peripheral neuropathy impaired patients with impaired glucose regulation (IGR). A total of 70 IGR patients received the conventional nerve conduction test, including 30 patients with peripheral neuropathy (PN) and 40 patients without peripheral neuropathy (NPN). The other 40 healthy individuals were recruited as controls. The serum TNF- $\alpha$ and IL-6 in IGR patients were higher than in control group, and serum TNF- $\alpha$ and IL-6 levels in IGR-PN group were higher than in IGR-NPN group $(27.7 \pm 17.8$ versus $13.1 \pm 6.7 \mathrm{pg} / \mathrm{mL}$ and $18.1 \pm 17.7$ versus $6.4 \pm 3.7 \mathrm{pg} / \mathrm{mL}$, resp., both $p<0.05)$. Multifactors logistic regression analysis showed that TNF- $\alpha(\mathrm{OR}=0.893$; $p=0.009$ ) was an independent factor affecting whether IGR could combine with peripheral neuropathy. TNF- $\alpha$ and IL- 6 could aggregate peripheral neuropathy in impaired glucose regulation patients; TNF- $\alpha$ might be independent risk factor for peripheral neuropathy in glucose regulation impaired patients.
\end{abstract}

\section{Introduction}

Abnormal glucose metabolism is one of the important reasons for the occurrence of peripheral neuropathy $[1,2]$. Some patients have symptoms of peripheral neuropathy before the diagnosis of their diabetes [3]. Most impaired glucose regulation (IGR) patients will eventually develop to diabetes and the relationship between IGR and peripheral neuropathy is still controversial $[4,5]$. Divisova et al. indicated that prediabetes and early type 2 diabetes mellitus are the significant risk factors of peripheral neuropathy [6]. Recently, a study showed that, based on the clinical symptoms results, $22 \%$ of diabetic patients and $23.9 \%$ of patients with abnormal glucose regulation of patients present distal symmetric multiple peripheral neuropathy [7]. These studies indicated that there were a large number of patients with diabetes and impaired glucose regulation who have peripheral neuropathy needed for early detection and intervention.
The mechanism of peripheral neuropathy has not yet been elucidated clearly. Compelling researches have indicated that the proinflammatory cytokines play important roles in the process of diabetic peripheral neuropathy. The proinflammatory cytokines such as tumor necrosis factor-alpha (TNF- $\alpha$ ) and interleukin-6 (IL-6) participate in the process of diabetic peripheral neuropathy [8]. The recent metaanalysis showed that type 2 diabetic patients with peripheral neuropathy have obviously increased serum TNF- $\alpha$ levels compared to type 2 diabetic patients without peripheral neuropathy and healthy controls, and high level of serum TNF- $\alpha$ may be associated with increased risk of peripheral neuropathy independently [9]. Tiftikcioglu et al. showed an increase in IL-6 in those with IGT-PN [10]. In this study, we measured serum level of TNF- $\alpha$ and IL- 6 in normal control (NC) and IGR patients with or without peripheral neuropathy to explore the mechanism of TNF- $\alpha$ and IL- 6 in the process of peripheral neuropathy. 


\section{Patients and Methods}

2.1. Patients and Ethnic Consideration. The study was approved and registered in the hospital ethics committee of the Tianjin Third Central Hospital in January 2014; the ethics committee approved relating screening, treatment, and data collection of these patients; all subjects signed written informed consent form. All works were undertaken following the provisions of the Declaration of Helsinki.

IGR patients were recruited from department of endocrinology and department of neurology. IGR was diagnosed according to the glucose regulation criteria of WHO in 1999 [11]. IGR includes impaired fasting glucose (IFG) and impaired glucose tolerance (IGT). $75 \mathrm{~g}$ oral glucose tolerance test (OGTT) was recommended for each patient; fasting plasma glucose (FPG) levels > $126 \mathrm{mg} / \mathrm{dL}$ constitute diabetes, whereas an intermediate designation IFG was established for FPG $>110 \mathrm{mg} / \mathrm{dL}$ but $<126 \mathrm{mg} / \mathrm{dL}$. A glucose level $>140 \mathrm{mg} / \mathrm{dL}$ but $<200 \mathrm{mg} / \mathrm{dL} 2 \mathrm{~h}$ after a $75 \mathrm{~g}$ OGTT was defined as IGT. The peripheral neuropathy was diagnosed according to the criteria of American Diabetes Association (ADA) in 2010 [12]; the clinical symptoms and signs and nerve conduction abnormalities in IGR-PN group were in accordance with the diagnosis of distal symmetrical polyneuropathy. A total of 70 IGR patients were consecutively enrolled into the study.

Patients with the following criteria were excluded: (1) patients with type 1 diabetes; (2) patients with serious liver or renal insufficiency; (3) patients with severe thyroid function; (4) patients with refractory hypertension; (5) pregnant patients; (6) patients with recent history of infection; (7) patients with cerebral vascular disease; (8) patients with history of spinal joint disease; (9) patients with toxic peripheral neuritis; (10) patients with infection of multiple neuritis; (11) patients with chronic alcoholism; (12) patients with vasculitis (13); patients with autoimmune disease; (14) patients with tumors; and (15) patients who have other diagnosed nondiabetic peripheral nerve lesions.

At the meantime, another 40 healthy volunteers were recruited from the hospital's physical examination center; all of them were conformed to normal oral glucose tolerance test (OGTT) results.

2.2. Demographic Data. The demographic data including height, weight, waist circumference, body mass index (BMI), and blood pressure were recorded in the 3 groups. 12 hours after fasting, their middle elbow vein blood was drawn. The blood glucose oxidase method was used for determination of fasting blood glucose (FBG). The high pressure liquid chromatography (HPLC) method was used for determination of their glycosylated hemoglobin (HbAlc). The enzyme chemical method was used for determination of triglycerides (TG), total cholesterol (TC), high-density lipoprotein cholesterol (HDL-C), and low-density lipoprotein cholesterol (LDL-C). The powder color turbidity method was used to measure high-sensitivity C-reactive protein (hs-CRP). Their serum TNF- $\alpha$ and IL- 6 levels were measured by chemiluminescence method (DPC1000, Siemens Healthcare Global, Erlangen, Germany).
2.3. $75 \mathrm{~g}$-OGTT Methods. All subjects have been fasting since 10:00 pm before the test. Fasting blood sample was collected by venipuncture. Subjects were given $200 \mathrm{~mL}$ water equivalent with $75 \mathrm{~g}$ anhydrous glucose. They consumed the glucose load within 5 minutes. Time of subjects finishing the glucose load was recorded on the fasting blood sample tube and on the clinical chemistry form as time 0 . Blood sample was taken at +120 minutes. Subjects were seated and remained within the department throughout test. Smoking and drinking tea or coffee were avoided. Prior to the OGTT of all of the subjects, restricted diet containing $>150$ g carbohydrate per day for 3 days was performed.

2.4. Conventional Nerve Conduction Detection. The motor conduction of median nerve, ulnar nerve, and common peroneal nerve (CPN) and the sensory conduction of median nerve, ulnar nerve, posterior tibial nerve (PTN), and sural nerve of all subjects were measured by the Dantec Keypoint Workstation (Suite, CA, USA). According to the revised criteria of Dyck et al. in 2011 [13], abnormal nerve conduction studies (NCS) were defined as one of the following criteria: prolonged incubation period; slowing of conduction velocity; reducing of CMAP and SNAP amplitude; or unsuccessful eliciting of certain arbitrary waveform.

2.5. Severity of Neuropathy. The severity of neuropathy was graded according to Toronto Clinical Scoring System (TCSS) [14]: $1-5$ points for no neuropathy; $6-8$ points for mild neuropathy; 9-11 points for moderate neuropathy; and 12-19 points for severe neuropathy. Symptom, reflex, and sensory tests, including pinprick, temperature, light touch, vibration, and position sensation, were performed as part of the TCSS.

2.6. Data Analysis. SPSS 18.0 software was used for analysis. Measurement data were expressed as mean \pm SD and compared with single-factor variance analysis; normal distributed measurement data were compared using $t$-test; variance of abnormal distributed data comparison was performed according to Dunnett's analysis; chi-square test was used for count data comparison. Correlation between variables was decided by Pearson's correlation analysis and logistic regression analysis was used for multivariate analysis.

\section{Results}

3.1. Demographic Data Comparison. There was no statistical difference $(p>0.05)$ in age and gender distribution between 3 groups (Table 1). BMI, systolic blood pressure, and diastolic blood pressure have no statistical difference $(p>0.05)$ between IGR-PN group and IGR-NPN group, while the waistline in IGR-PN group was significantly greater than that in IGR-NPN group $(p<0.05)$.

3.2. Laboratory Parameter Comparison. As we can see from Tables 2 and 3, compared with normal control group, the FBG, OGTT-2h glucose, HbAlc, TG, IL-6, and TNF- $\alpha$ in IGR-PN group and IGR-NPN group were significantly increased $(p<0.05)$. The TC level in the IGR-NPN group was 
TABLE 1: Demographic data comparison in 3 groups.

\begin{tabular}{lccc}
\hline & $\begin{array}{c}\text { Normal } \\
\text { control }\end{array}$ & IGR-NPN & IGR-PN \\
\hline Case number & 40 & 40 & 30 \\
Gender $(\mathrm{M} / \mathrm{F})$ & $20 / 20$ & $20 / 20$ & $15 / 15$ \\
Age $(\mathrm{yr})$ & $58.9 \pm 8.6$ & $57.7 \pm 9.2$ & $59.8 \pm 10.4$ \\
BMI $\left(\mathrm{kg} / \mathrm{m}^{2}\right)$ & $22.7 \pm 1.2$ & $24.8 \pm 3.4$ & $25.7 \pm 3.9$ \\
Waistline $(\mathrm{cm})$ & $68.6 \pm 6.8$ & $86.3 \pm 8.4^{*}$ & $88.9 \pm 10.9^{*, \#}$ \\
Systolic pressure $(\mathrm{mmHg})$ & $122.1 \pm 8.6$ & $132.4 \pm 14.4$ & $133.5 \pm 13.3$ \\
$\begin{array}{l}\text { Diastolic pressure } \\
(\mathrm{mmHg})\end{array}$ & $72.2 \pm 7.2$ & $82.4 \pm 9.0$ & $84.7 \pm 11.6$ \\
\hline${ }^{*} p<0.05$, compared with normal control; ${ }^{*} p<0.05$, compared with IGR- \\
NPN group.
\end{tabular}

TABLE 2: Laboratory parameter comparison in 3 groups.

\begin{tabular}{lccc}
\hline & Normal control & IGR-NPN & IGR-PN \\
\hline FBG (mmol/L) & $5.4 \pm 0.3$ & $6.2 \pm 0.8^{*}$ & $6.2 \pm 0.9^{*}$ \\
OGTT-2 h glucose & $6.5 \pm 0.7$ & $9.3 \pm 1.3^{*}$ & $8.9 \pm 1.9^{*}$ \\
(mmol/L) & $5.2 \pm 0.5$ & $6.2 \pm 0.5^{*}$ & $6.2 \pm 0.3^{*}$ \\
HbAlc $(\%)$ & $1.1 \pm 0.3$ & $1.6 \pm 1.3^{*}$ & $1.7 \pm 0.9^{*}$ \\
TG (mmol/L) & $4.7 \pm 0.3$ & $5.2 \pm 1.1^{*}$ & $5.0 \pm 0.9$ \\
TC (mmol/L) & $1.3 \pm 0.1$ & $1.3 \pm 0.3$ & $1.3 \pm 0.2$ \\
HDL (mmol/L) & $2.8 \pm 0.2$ & $3.0 \pm 0.7$ & $2.8 \pm 0.6$ \\
\hline LDL (mmol/L) & &
\end{tabular}

${ }^{*} p<0.05$, compared with normal control.

TABLE 3: IL-6 and TNF- $\alpha$ levels in each group.

\begin{tabular}{lccc}
\hline & Normal control & IGR-NPN & IGR-PN \\
\hline IL-6 $(\mathrm{pg} / \mathrm{mL})$ & $2.7 \pm 1.0$ & $6.4 \pm 3.7^{*}$ & $18.1 \pm 17.7^{*, \#}$ \\
TNF- $\alpha(\mathrm{pg} / \mathrm{mL})$ & $4.2 \pm 1.5$ & $13.1 \pm 6.7^{*}$ & $27.7 \pm 17.8^{*, \#}$ \\
CRP $(\mathrm{mmol} / \mathrm{L})$ & $3.1 \pm 1.4$ & $3.0 \pm 1.7$ & $4.3 \pm 4.7$ \\
\hline
\end{tabular}

${ }^{*} p<0.05$, compared with normal control; ${ }^{\#} p<0.05$, compared with IGRNPN group.

significantly higher than in the NC group $(p<0.05)$, while there was no significant difference compared with the IGR$\mathrm{PN}(p>0.05)$. There was no statistical difference $(p>0.05)$ in HDL-C and LDL-C distribution between 3 groups $(p>$ 0.05). There were no statistical differences in FBG, OGTT-2 $\mathrm{h}$ glucose, HbAlc, and TG between IGR-PN group and IGRNPN group ( $p>0.05)$; but IL-6 and TNF- $\alpha$ levels in IGRPN group were significantly higher than those in IGR-NPN group $(p<0.05)$. The level of CRP in IGR-PN group was higher than that in the NC group and IGR-NPN group in this study, but there was no statistical difference between IGRPN group and IGR-NPN group $(p>0.05)$, which might be caused by insufficient sample size.

3.3. Neuropathy Comparison. Nerve conduction velocities in IGR-PN group were compared with IGR-NPN group in Table 5. Motor nerve conduction velocities (MNCV) in common peroneal nerve and sensory nerve conduction
TABLE 4: Neuropathy score comparison in 3 groups.

\begin{tabular}{lccc}
\hline & Normal control & IGR-NPN & IGR-PN \\
\hline Case number & 40 & 40 & 30 \\
Symptom scores & $0.05 \pm 0.22$ & $0.05 \pm 0.22$ & $0.33 \pm 0.48^{\#}$ \\
Reflex scores & $0.18 \pm 0.45$ & $0.68 \pm 0.76^{*}$ & $5.03 \pm 1.27^{*, \#}$ \\
Sensory scores & $0.30 \pm 0.60$ & $0.78 \pm 0.73^{*}$ & $2.83 \pm 1.29^{*, \#}$ \\
TCSS (score) & $0.52 \pm 1.01$ & $1.52 \pm 1.38^{*}$ & $8.01 \pm 2.40^{*, \#}$ \\
${ }^{*} p<0.05$, compared with normal control; ${ }^{\#} p<0.05$, compared with IGR- \\
NPN group.
\end{tabular}

TABLE 5: Nerve conduction velocity in patients with impaired glucose regulation.

\begin{tabular}{lccc}
\hline NCV $(\mathrm{m} / \mathrm{s})$ & IGR-NPN & IGR-PN & $p$ \\
\hline Motor & & & \\
$\quad$ Median & $57.90 \pm 4.06$ & $57.98 \pm 4.67$ & 0.941 \\
Ulnar & $68.97 \pm 7.48$ & $67.42 \pm 6.86$ & 0.376 \\
CPN & $49.67 \pm 4.64$ & $47.37 \pm 4.78$ & $0.046^{*}$ \\
Sensory & & & \\
Median & $49.21 \pm 8.94$ & $47.08 \pm 8.39$ & 0.315 \\
Ulnar & $57.90 \pm 6.05$ & $55.94 \pm 6.94$ & 0.213 \\
PTN & $47.60 \pm 7.56$ & $40.57 \pm 6.06$ & $0.010^{*}$ \\
Sural & $56.35 \pm 5.76$ & $53.88 \pm 6.89$ & 0.107 \\
\hline${ }^{*} p<0.05$ is significant. & &
\end{tabular}

TABLE 6: Correlation of TNF- $\alpha$ with nerve conduction velocity in patients with IGR-PN.

\begin{tabular}{lcc}
\hline Nerve conduction velocity & $r$ & $p$ \\
\hline Motor & & \\
Median & -0.266 & 0.155 \\
Ulnar & -0.322 & 0.125 \\
CPN & -0.195 & 0.301 \\
Sensory & & \\
Median & -0.330 & 0.075 \\
Ulnar & -0.244 & 0.194 \\
PTN & -0.144 & 0.448 \\
Sural & -0.291 & $0.015^{*}$ \\
\hline
\end{tabular}

$r$ is Pearson's coefficient. ${ }^{*} p<0.05$ is significant.

velocities (SNCV) in posterior tibial nerve were significantly decreased $(p<0.05)$, while all other nerve velocities difference were not significant. Amplitudes in IGR-PN group were compared with IGR-NPN group in Table 7. CMAP amplitude in common peroneal nerve and SNAP amplitude in median nerve and posterior tibial nerve were significantly decreased $(p<0.05)$, while all other nerve amplitudes differences were not significant. Correlation of biomarker TNF- $\alpha$ with nerve conduction studies in IGR-PN group showed significant negative correlation with sural SNCV (Table 6) and posterior tibial nerve SNAP amplitude (Table 8).

The neuropathy scores in IGR-PN group and IGR-NPN group were significantly higher than in normal control group $(p<0.05$, Table 4). Further comparison demonstrated that the neuropathy scores in IGR-PN group were significantly 
TABLE 7: Nerve amplitude in patients with impaired glucose regulation.

\begin{tabular}{lccc}
\hline Amplitude & IGR-NPN & IGR-PN & $p$ \\
\hline CMAP amplitude $(\mathrm{mV})$ & & & \\
Median & $10.48 \pm 2.22$ & $9.86 \pm 2.51$ & 0.281 \\
Ulnar & $10.18 \pm 1.47$ & $10.09 \pm 1.67$ & 0.810 \\
CPN & $5.54 \pm 1.62$ & $4.42 \pm 1.51$ & $0.004^{*}$ \\
SNAP amplitude $(\mu \mathrm{V})$ & & & \\
Median & $19.89 \pm 9.07$ & $15.17 \pm 7.48$ & $0.023^{*}$ \\
Ulnar & $9.855 \pm 3.29$ & $8.55 \pm 3.52$ & 0.115 \\
PTN & $1.89 \pm 0.97$ & $1.29 \pm 0.67$ & $0.005^{*}$ \\
Sural & $10.05 \pm 1.66$ & $9.56 \pm 1.65$ & 0.223 \\
\hline
\end{tabular}

${ }^{*} p<0.05$ is significant.

TABLE 8: Correlation of TNF- $\alpha$ with nerve amplitude in patients with IGR-PN.

\begin{tabular}{lcc}
\hline Amplitude & $r$ & $p$ \\
\hline CMAP amplitude $(\mathrm{mV})$ & & \\
Median & -0.074 & 0.544 \\
Ulnar & -0.063 & 0.607 \\
CPN & -0.036 & 0.769 \\
SNAP amplitude $(\mu \mathrm{V})$ & & \\
Median & -0.148 & 0.222 \\
Ulnar & -0.213 & 0.077 \\
PTN & -0.288 & $0.016^{*}$ \\
Sural & -0.016 & 0.934 \\
\hline
\end{tabular}

$r$ is Pearson's coefficient. ${ }^{*} p<0.05$ is significant.

higher than those in IGR-NPN group with significant difference $(p<0.05)$.

3.4. Correlation Analysis. In this study, whether impaired glucose regulation combined with peripheral neuropathy was considered as the dependent variable or the FBG, OGTT$2 \mathrm{~h}$ glucose, HbAlc, TG, TC, HDL-C, LDL-C, CRP, IL-6, and TNF- $\alpha$ were considered as the independent variable for logistic regression analysis, the results showed that TNF- $\alpha$ $(\mathrm{OR}=0.893 ; p=0.009)$ was independent factor affecting whether impaired glucose regulation patient could have peripheral neuropathy. Further, Pearson's correlation analysis showed that TNF- $\alpha$ levels significantly positively related with TCSS score $(r=0.331 ; p=0.005)$ (Figure 1).

\section{Discussions}

Abnormal glucose metabolism is the most common cause of peripheral neuropathy. Compelling studies have confirmed that immune factors are involved in the occurrence of diabetic peripheral neuropathy [15-17] and immune cytokines play important role in this process. However, the pathogenesis of impaired glucose regulation combined with peripheral neuropathy is not clear. In this study, we found that the serum levels of TNF- $\alpha$ and IL-6 in impaired glucose regulation (IGR) patients with peripheral neuropathy increased

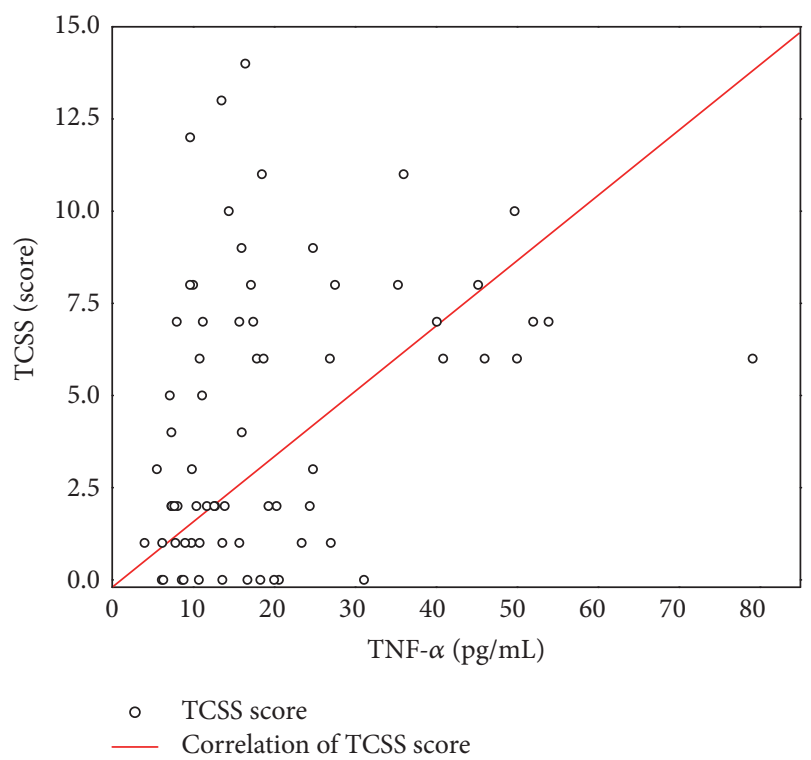

FIGURE 1: The correlation of TCSS score and TNF- $\alpha$ in IGR patients. $r=0.331 ; p=0.005$.

obviously, and Pearson's correlation analysis confirmed that TNF- $\alpha$ and TCSS were significantly positively correlated with whether IGR patients could have peripheral neuropathy. Our research suggested that immune factors, especially TNF- $\alpha$, are involved with IGR peripheral neuropathy pathogenesis. In the present study, level of TNF- $\alpha$ in IGR-PN is correlated with sensory nerve. This result is supported by previous study, where significant correlation of TNF- $\alpha$ with sensory nerve conduction velocity was shown [18]. This finding also coincides with result reported by Sumner et al. and Drory et al., where it was shown that nerve function may be affected by this cytokine level $[19,20]$.

TNF- $\alpha$ is a bioactive factor activated by monocytes/ macrophages, natural killer (NK) cells, B cells, and fat cells $[21,22]$. The long-term hyperglycemia could cause nerve myelin protein glycosylation, which was recognized by specific macrophage later. And then the glycosylated myelin protein was phagocytosed by the macrophage. This may cause the nerve demyelination and myelin antigens to be exposed. Activated by these antigens, $\mathrm{T}$ cells produce a series of cytokines (including TNF- $\alpha$ ) which raised the autoimmune response and mediated inflammatory reaction [23]. TNF$\alpha$ involvement in the regulation of chronic inflammation, immune response, and multiple metabolic disorders promote the proliferation of $\mathrm{T}$ cells to stimulate cytokine production [24]. In addition, TNF- $\alpha$ has toxic effects on oligodendrocytes, can cause demyelination, and could stimulate mononuclear cells and endothelial cells secreting cytokines such as IL$1 \beta$ and IL- 6 and thus enhance the amplification effect of itself directly or indirectly [25].

In addition, TNF- $\alpha$ could inhibit endothelial nitric oxide synthase (NOS) activity, reduce the vasodilation effect induced by $\mathrm{NO}$, and promote the expression of a variety of growth factors and cell adhesion molecules and thus causes 
endothelial dysfunction and lead to thickening of the capillary basement membrane, swelling, hyperplasia, and hyaline degeneration of endothelial cell and finally causes the luminal stenosis, hemodynamic abnormalities, hypoperfusion, and damaging the nerve and its nutrient vessels [26].

A research performed by Yamakawa et al. demonstrated that after knocking out the TNF- $\alpha$ gene, the diabetic peripheral neuropathy was not developed in mice. The disappearance of TNF- $\alpha$ gene to completely prevent the nerve lesions of STZ induced diabetic mouse. In addition, inactivating the expression of monoclonal antibodies of TNF- $\alpha$ and inhibiting the expression of mRNA could play a role in the treatment of diabetic peripheral neuropathy [27]. Other studies have also demonstrated that TNF- $\alpha$ is important inflammatory cytokine in the treatment of diabetic peripheral neuropathy $[28,29]$. Studies have also shown that serum TNF- $\alpha$ mediates IGT associated insulin resistance; the serum TNF- $\alpha$ levels in IGT patients were significantly increased and were positively related with waist circumference, high-density lipoprotein, and triglycerides [30].

TCSS is a synthesis grading system formulated based on the characteristics of DPN with advantage of being simple and comprehensive (including evaluating the symptoms and signs of subject). TCSS is suitable for large-scale epidemiological survey and evaluation of DPN severity in clinical practice. Studies have reported that the TCSS score had a moderate consistency with the nerve conduction velocity (NCV); the correlation between TCSS grading of neuropathy and NCV was better [31]. In consistency with Katulanda's result, our study shows that TNF- $\alpha$ levels have a significant positive correlation with TCSS, and TNF- $\alpha$ is an independent risk factor for peripheral neuropathy in impaired glucose regulation patients.

\section{Conclusion}

In conclusion, TNF- $\alpha$ is an independent risk factor of peripheral neuropathy in IGR patients besides hyperglycemia, blood pressure, and lipid. It may have potential clinical value in the prediction of early diabetic peripheral neuropathy. So far, there is still lack of early and effective diagnosis method for prediabetes peripheral nerve lesions in clinical practice, and prediabetic peripheral neuropathy clinical trials also lack a sensitive indicator for early detection. We hope that further basic and clinical research could provide an objective basis for early diagnosis of prediabetes peripheral neuropathy through the detection of serum TNF- $\alpha$ level so as to conduct the early prevention and treatment of peripheral neuropathy complications.

\section{Competing Interests}

The authors declare that there are no competing interests.

\section{Acknowledgments}

The study was supported by research grants from Key Projects of Health Industry of Tianjin Health Department (14KG110).

\section{References}

[1] H. Ince, H. A. Taşdemir, M. Aydin, H. Ozyürek, and H. E. Tilki, "Evaluation of nerve conduction studies in obese children with insulin resistance or impaired glucose tolerance," Journal of Child Neurology, vol. 30, no. 8, pp. 989-999, 2015.

[2] K. Pourhamidi, L. B. Dahlin, E. Englund, and O. Rolandsson, "Evaluation of clinical tools and their diagnostic use in distal symmetric polyneuropathy," Primary Care Diabetes, vol. 8, no. 1, pp. 77-84, 2014.

[3] C. Ly, "Research on the progress of diabetic peripheral neuropathy," The Chinese Neurologist Magazine, vol. 39, pp. 433-438, 2006.

[4] L. A. Zilliox, S. K. Ruby, S. Singh, M. Zhan, and J. W. Russell, "Clinical neuropathy scales in neuropathy associated with impaired glucose tolerance," Journal of Diabetes and Its Complications, vol. 29, no. 3, pp. 372-377, 2015.

[5] S. Lupachyk, P. Watcho, A. A. Obrosov, R. Stavniichuk, and I. G. Obrosova, "Endoplasmic reticulum stress contributes to prediabetic peripheral neuropathy," Experimental Neurology, vol. 247, pp. 342-348, 2013.

[6] S. Divisova, E. Vlckova, M. Hnojcikova et al., "Prediabetes/early diabetes-associated neuropathy predominantly involves sensory small fibres," Journal of the Peripheral Nervous System, vol. 17, no. 3, pp. 341-350, 2012.

[7] B. W. C. Bongaerts, W. Rathmann, M. Heier et al., "Older subjects with diabetes and prediabetes are frequently unaware of having distal sensorimotor polyneuropathy: The KORA F4 study," Diabetes Care, vol. 36, no. 5, pp. 1141-1146, 2013.

[8] M. H. Rahman, M. K. Jha, and K. Suk, "Evolving insights into the pathophysiology of diabetic neuropathy: implications of malfunctioning glia and discovery of novel therapeutic targets," Current Pharmaceutical Design, vol. 22, no. 6, pp. 738-757, 2016.

[9] Z.-P. Mu, Y.-G. Wang, C.-Q. Li et al., "Association between tumor necrosis factor- $\alpha$ and diabetic peripheral neuropathy in patients with type 2 diabetes: a meta-analysis," Molecular Neurobiology, 2016.

[10] B. I. Tiftikcioglu, T. Duksal, S. Bilgin, S. Kose, and Y. Zorlu, "Association between the levels of IL-6, sE-selectin and distal sensory nerve conduction studies in patients with prediabetes," European Neurology, vol. 75, no. 3-4, pp. 124-131, 2016.

[11] K. G. Alberti and P. Z. Zimmet, "Definition, diagnosis and classification of diabetes mellitus and its complications-part 1: diagnosis and classification of diabetes mellitus. Provisional report of a WHO consultation," Diabetic Medicine, vol. 15, no. 7, pp. 539-553, 1998.

[12] S. Tesfaye, A. J. M. Boulton, P. J. Dyck et al., "Diabetic neuropathies: update on definitions, diagnostic criteria, estimation of severity, and treatments," Diabetes Care, vol. 33, no. 12, pp. 2285-2293, 2010.

[13] P. J. Dyck, R. E. Carter, and W. J. Litchy, "Modeling nerve conduction criteria for diagnosis of diabetic polyneuropathy," Muscle and Nerve, vol. 44, no. 3, pp. 340-345, 2011.

[14] S. Sachedina and C. Toth, "Association of comorbidities with increasing severity of peripheral neuropathy in diabetes mellitus," World Journal of Diabetes, vol. 4, no. 4, pp. 135-144, 2013.

[15] X. Zhang, Y. Hu, J. Shen et al., "Low levels of ficolin-3 are associated with diabetic peripheral neuropathy," Acta Diabetologica, vol. 53, pp. 295-302, 2016.

[16] M. Kazamel and P. J. Dyck, "Sensory manifestations of diabetic neuropathies: anatomical and clinical correlations," Prosthetics and Orthotics International, vol. 39, no. 1, pp. 7-16, 2015. 
[17] T. Kanda, "Biology of the blood-nerve barrier and its alteration in immune mediated neuropathies," Journal of Neurology, Neurosurgery and Psychiatry, vol. 84, no. 2, pp. 208-212, 2013.

[18] M. Matsuda, F. Kawasaki, H. Inoue et al., "Possible contribution of adipocytokines on diabetic neuropathy," Diabetes Research and Clinical Practice, vol. 66, supplement 1, pp. S121-S123, 2004.

[19] C. J. Sumner, S. Sheth, J. W. Griffin, D. R. Cornblath, and M. Polydefkis, "The spectrum of neuropathy in diabetes and impaired glucose tolerance," Neurology, vol. 60, no. 1, pp. 108111, 2003.

[20] V. E. Drory, D. Lev, G. B. Groozman, M. Gutmann, and J. M. Klausner, "Neurotoxicity of isolated limb perfusion with tumor necrosis factor," Journal of the Neurological Sciences, vol. 158, no. 1, pp. 1-4, 1998.

[21] V. S. Soumyarani and N. Jayakumari, "Oxidatively modified high density lipoprotein promotes inflammatory response in human monocytes-macrophages by enhanced production of ROS, TNF- $\alpha$, MMP-9, and MMP-2," Molecular and Cellular Biochemistry, vol. 366, no. 1-2, pp. 277-285, 2012.

[22] C. Olsnes, H. Stavang, J. Olofsson, and H. J. Aarstad, “TNF- $\alpha$ is secreted by monocytes in transit to become macrophages, but not by peripheral blood monocytes, following OK-432 (Lyophilized S. pyogenes) stimulation," Scandinavian Journal of Immunology, vol. 66, no. 6, pp. 684-693, 2007.

[23] D. A. Joyce, G. Gimblett, and J. H. Steer, "Targets of glucocorticoid action on TNF- $\alpha$ release by macrophages," Inflammation Research, vol. 50, no. 7, pp. 337-340, 2001.

[24] L. C. R. Silva, L. C. M. Ortigosa, and G. Benard, "Anti-TNF$\alpha$ agents in the treatment of immune-mediated inflammatory diseases: mechanisms of action and pitfalls," Immunotherapy, vol. 2, no. 6, pp. 817-833, 2010.

[25] A. Fromont, J. De Seze, M. C. Fleury, J. F. Maillefert, and T. Moreau, "Inflammatory demyelinating events following treatment with anti-tumor necrosis factor," Cytokine, vol. 45 , no. 2 , pp. 55-57, 2009.

[26] J. S. Estrella, R. N. Nelson, B. K. Sturges et al., "Endoneurial microvascular pathology in feline diabetic neuropathy," Microvascular Research, vol. 75, no. 3, pp. 403-410, 2008.

[27] I. Yamakawa, H. Kojima, T. Terashima et al., "Inactivation of TNF- $\alpha$ ameliorates diabetic neuropathy in mice," American Journal of Physiology -Endocrinology and Metabolism, vol. 301, no. 5, pp. E844-E852, 2011.

[28] V. R. Drel, S. Lupachyk, H. Shevalye et al., "New therapeutic and biomarker discovery for peripheral diabetic neuropathy: PARP inhibitor, nitrotyrosine, and tumor necrosis factor- $\alpha$," Endocrinology, vol. 151, no. 6, pp. 2547-2555, 2010.

[29] T. Zhu, Q. Meng, J. Ji, X. Lou, and L. Zhang, “Toll-like receptor 4 and tumor necrosis factor-alpha as diagnostic biomarkers for diabetic peripheral neuropathy," Neuroscience Letters, vol. 585, pp. 28-32, 2015.

[30] N. C. Olson, P. W. Callas, A. J. G. Hanley et al., "Circulating levels of TNF- $\alpha$ are associated with impaired glucose tolerance, increased insulin resistance, and ethnicity: the insulin resistance atherosclerosis study," The Journal of Clinical Endocrinology \& Metabolism, vol. 97, no. 3, pp. 1032-1040, 2012.

[31] P. Katulanda, P. Ranasinghe, R. Jayawardena, G. R. Constantine, M. H. R. Sheriff, and D. R. Matthews, "The prevalence, patterns and predictors of diabetic peripheral neuropathy in a developing country," Diabetology and Metabolic Syndrome, vol. 4, no. 1, article 21, 2012. 


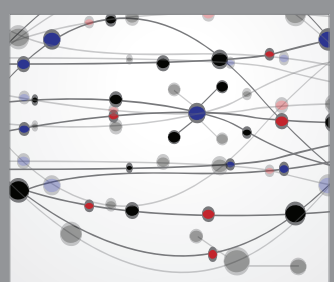

The Scientific World Journal
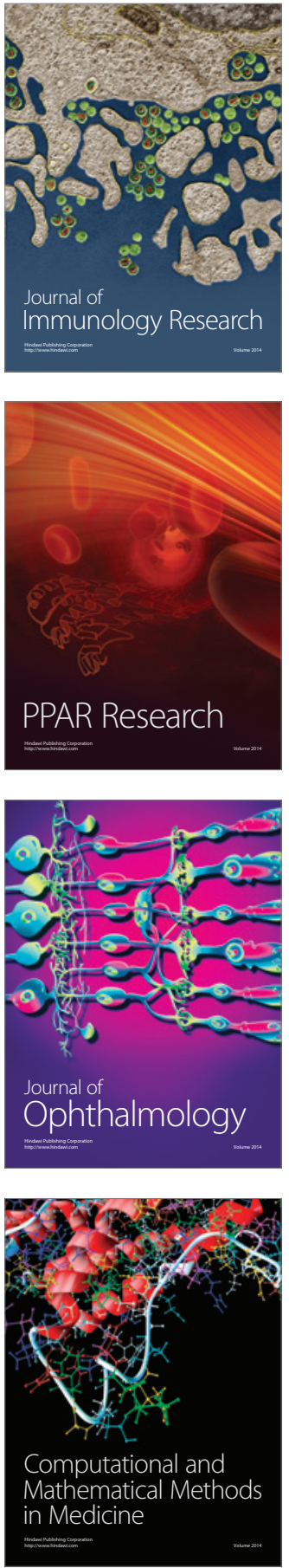

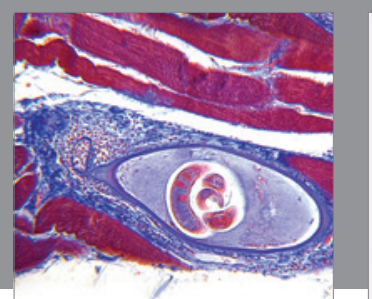

Gastroenterology Research and Practice
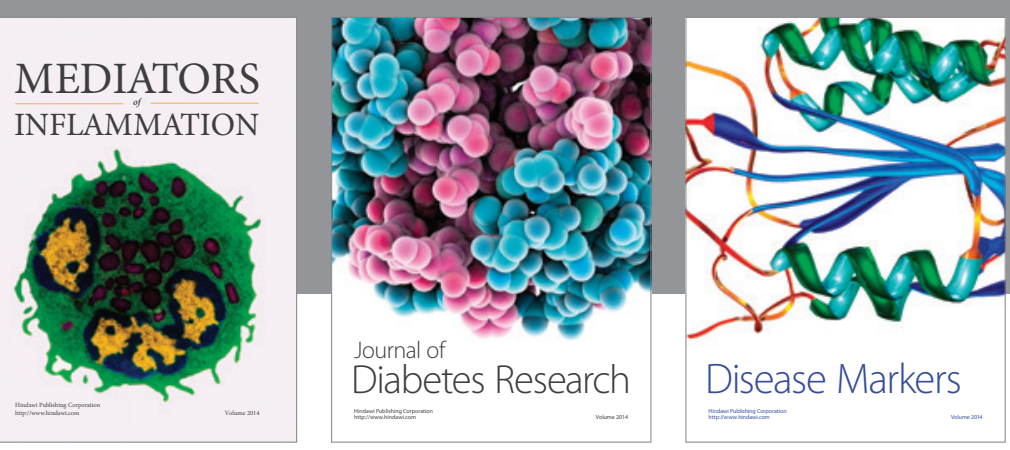

Disease Markers

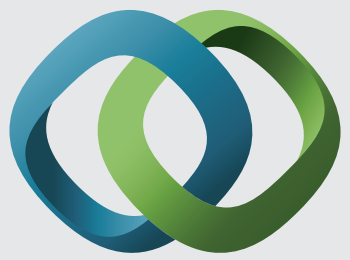

\section{Hindawi}

Submit your manuscripts at

https://www.hindawi.com
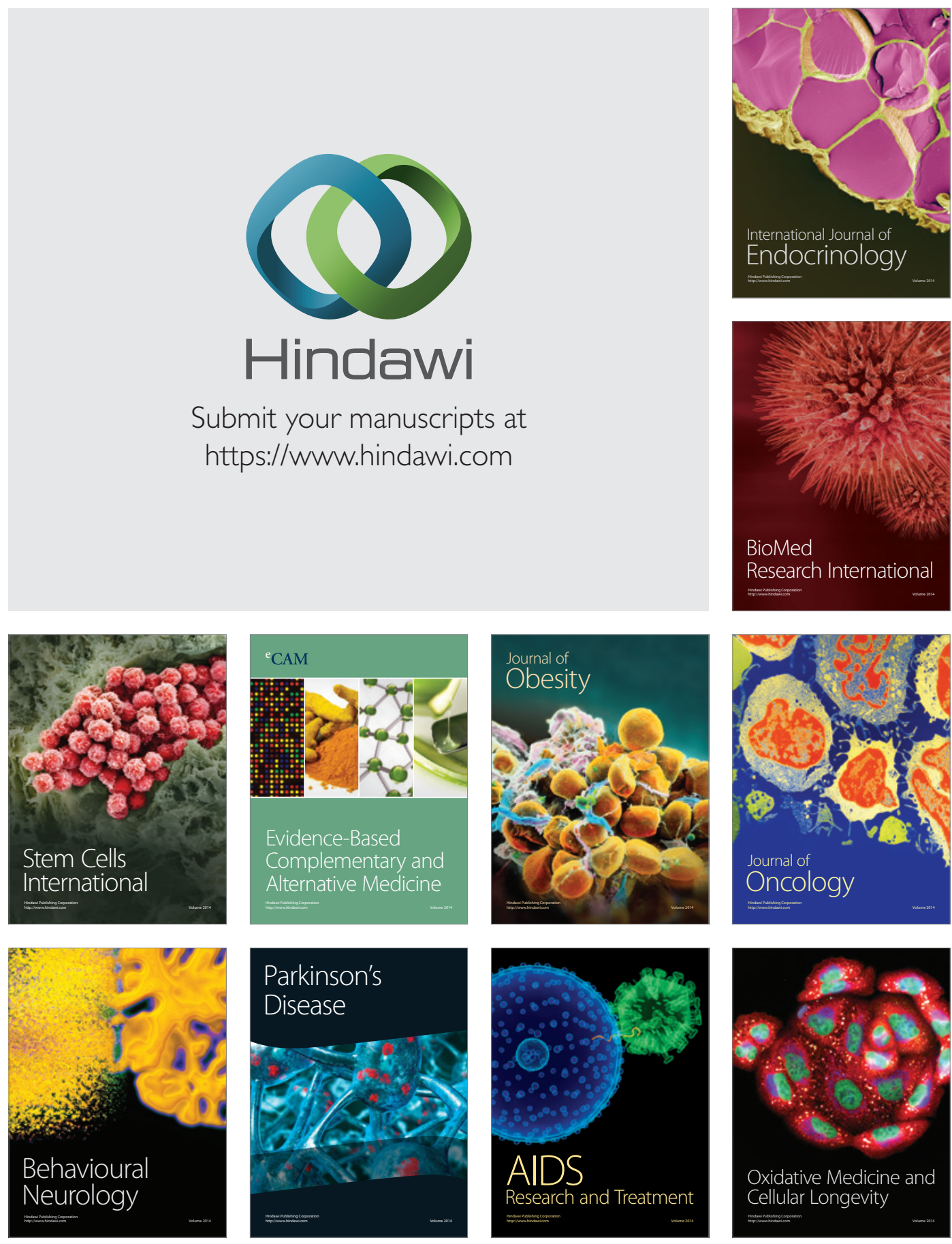